\title{
OBSTACLE DETECTION USING MONO VISION CAMERA AND LASER SCANNER
}

\author{
Sandip Mahajan ${ }^{1}$, Ravindra Bhosale ${ }^{2}$, Parag Kulkarni ${ }^{3}$ \\ ${ }^{I}$ Professor, IT Department, RGI Ahmednagar, Maharashtra, India, mahajan.sandip007@gmail.com \\ ${ }^{2}$ Professor, IT Department, RGI Ahmednagar, Maharashtra, India, bhosalerb2007@gmail.com \\ ${ }^{3}$ Professor, Computer Department, RGI Pune, Maharashtra, India, paragindia@gmail.com
}

\begin{abstract}
Single sensor is used to detect the obstacles but that sensor must have support of other sensor for confirmation of obstacles. Only one sensor is capable to take the decision but multi sensor decision is more precise and accurate. In this proposed system, mono vision camera and laser scanner is used to detect the obstacles for vehicle. In the system firstly laser scanner is used to detect the obstacles and then mono vision camera is used to confirmation of obstacles. Using mono vision camera we can detect the length and breadth of the obstacles and using laser scanner is used to detect the distance between our vehicle and obstacles. Depending on above data the system will detect the collision time. The proposed system is perfect example of cooperative fusion between multi sensor systems. The proposed system is generating very less false ration so that this system can be used in the real time.
\end{abstract}

Index Terms: Multi sensor data fusion, Laser Scanner, Mono Vision Camera, Contour analysis algorithm, Intelligent Vehicle, Obstacle Detection

\section{INTRODUCTION}

Perceptive systems are very powerful and widely used system in ADAS (Advanced Driving Assistance System). This type of systems are widely used for various subsystems particularly obstacle detection, lane change assistance etc. Obstacle detection is necessary for pre-crash, collision mitigation, stop and go, obstacle avoidance or inter distance management. Only one sensor is capable to detect the obstacle but also having high false rate. To avoid the errors we can use multiple sensors to detect the obstacle accurately and very low false rate. Currently many vehicles use ADAS systems for examples, reverse car assistance system, obstacle detection etc. mostly high values vehicle manufacture uses this type of systems for driving assistance purpose.

Obstacle Detection is a widely used and essential task for intelligent vehicles. This type of system can be implemented in real time, robustly and accurately without minimum false rate. Basic aim of this system is to identify the obstacles present on the road with better accuracy with additional information like height, width and depth of the obstacles. Depending on above information we can classify the obstacles whether they are pedestrian, car, truck, motorbike etc. Basic concept of proposed system is to use multi sensor devices to detect the obstacles present on the road and according to the distance between the vehicle and obstacle it gives the warning to the driver. In this system we use Laser
Scanner and Mono Vision Camera as sensors. Laser Scanner is used for the obstacle detection and mono vision camera is used for the confirmation of obstacles. In particular, using mono vision camera and a laser scanner seems to be an efficient solution. To take advantages of first sensor and avoid disadvantages using second sensor this type of configuration will increase the performance of system. The proposed system is having such type of innovative fusion scheme. In this system, the obstacle detection and tracking tasks are find out by the laser scanner. The mono vision camera is used subsequently to confirm the detections.

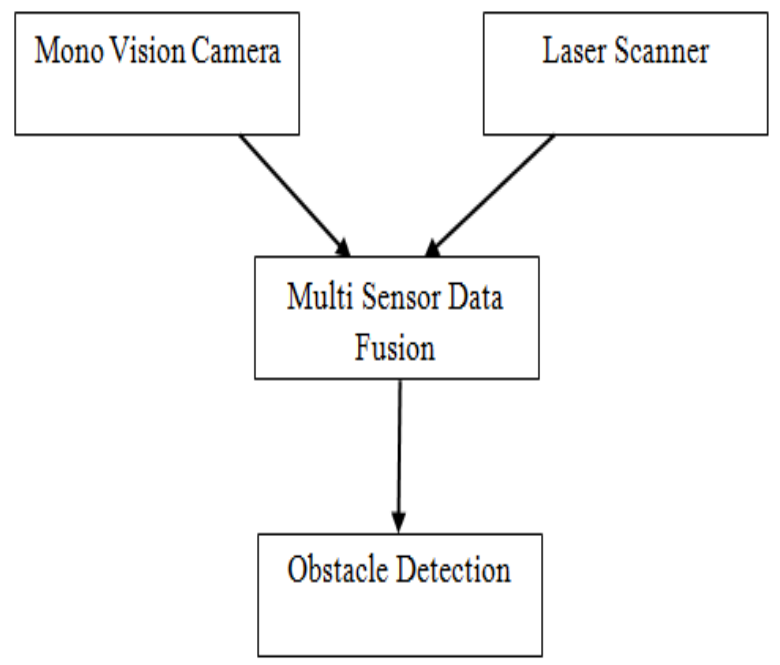

Fig. 1 General architecture of proposed system 


\section{LITERATURE SURVEY}

Obstacle detection us a powerful activity for autonomous systems in particular with ITS (Intelligent Transport Systems), Vehicles can be considered as a robots. The development of ADAS such as pre-crash, collision avoidance, collision migration or automatic cruise control requires that reliable road obstacle detection systems are available. There are a various approaches for detection of obstacles depending on the different types of sensors.

Obstacle can be detected by mono vision camera; this concept was kept by Franke and Joos in 2000. Depending on mono vision camera some algorithms are developed, so that they can provide robustly and with real time performance. The surface of the road including pitch, roll and non-flat geometry and then extract objects with respect to the estimated surface height and width of the objects can be evaluated. Road can be detected using a vision based lane detector and objects which are out of the road can be removed. However Laser Scanner data is used for the finding out the position and width of the obstacle. Multi sensor data fusion is used for the collection data from various types of sensor. Each sensor gives the information of same environment but in the different way. Collection of these data and find out the decision on current situation is gives the accurate and better solution.

Up till now client/server computing models is used in Distributed Sensor Networks (DSNs) to handle multi sensor data fusion. Sensor Technology and Computer Hard wares allow to deployment a large volume of data and smaller sensors in real time scenario. Multi Sensor Data Fusion term deals with the problems of fusion of data collected by multiple sensors together with knowledge in order to make accurate decision of real time environment. These types of systems are used for the Intelligent Transport Systems, Traffic Control, Maintaining Engineering, Remote Sensing, Robotics, Environmental Monitoring and Global Awareness etc. In 1960s, mathematical model for data fusion was appeared in the literature. This model was developed in the US. Afterwards, in 1970s data fusion approach was used for the Robotics and Defence purpose. Data Fusion Sub-Panel of the Joint Directors of Laboratories (JDL) was established by the US Department of Defence in 1986.This sub-Panel was used to address some of the main issues in data fusion and chart the new field related to data fusion terminology and procedures. At present data fusion techniques use in various applications like maintenance engineering, robotics, pattern recognition and radar tracking, mine detection and other military applications, remote sensing, traffic control, aerospace systems, law enforcement, medicine, finance, metrology, and geo-science.
Basic aim of ADAS is the collect the information from environment and driver. Like environmental information like drive straight, turning, overtaking information, meeting pedestrian etc. and driver information like observant, sleepy, drowsy, aggressive, using mobile etc. The combination of these parameters will be used to reflect a proper diving situation encountered by the driver. So we need an optimal selection of some appropriate sensors to monitor all these factors. There are various sensors used in the ADAS purpose like RADAR or Laser Scanner, Mono Vision camera or Mono Vision Camera. Radar shows limited lateral spatial information because it is not available at all, the field of view is narrow, or the resolution is reduced at large distances. Another problem with RADAR is low ability of discrimination, clustering error, and recognition latency. These all problem is solve using Laser Scanner .Instead of Mono vision camera we can use CCD camera and CMOS camera, but both the alternative having disadvantages, these disadvantages is overcame by using mono vision camera. CCD camera technology limits the frequency of the detection to the video frame rate $(25 \mathrm{~Hz})$ detection failures can be observed for blooming effects, due to sun or vehicle lights in the night conditions. On the other side CMOS camera produce noisy data. This may cause error and false alarm maybe raised when the road is non-constant roll. Mono vision camera can detect the obstacle up to few meters, because of the limited detection range. Usually fusion algorithms in the road context use homogeneous data concerning obstacles position obtained from Laser scanner and hardly take into account complementary features to enhance the detection results.

\section{PROPOSED SYSTEM}

Proposed system is a multi-sensor data fusion approach between the Laser scanner and mono vision camera in order to take advantage of the best features and cope with the drawbacks of sensors to perform robust, accurate and real time. Obstacle detection in the vehicle to be exploited for driving assistance purpose, a system must have low false detection rate with this idea in mind we propose a system with Laser scanner and mono vision camera for road obstacle detection. The obstacles are tracked by the Laser scanner and then mono vision camera is used for the confirmation of obstacles on the road.

Laser scanner and mono vision camera are complementary sensors, after fusion the final position of the detected obstacles is provided by Laser scanner, which is more precise and accurate than the mono vision camera. Laser scanner provides the width and depth of the obstacles whereas height of the obstacles is given by the mono vision 
camera. Laser scanner some time provide false data if laser hits to road pitch. Mono vision camera is also capable of finding the obstacle by using contour detection algorithm, but it cannot find the distance between the vehicle and obstacle. One sensor is not capable of taking a decision, so that multiple sensors are used to take decision. This decision is more accurate and precise. Proposed system is cooperative fusion between Laser Scanner and Mono vision camera in order to minimize the drawbacks of sensors by the other sensors and generate the result robust, accurate and real time. In this system Laser scanner is used to detect the different obstacles with their distance between vehicle and obstacle. Mono vision camera is used to give the confirmation of multi obstacles. Proposed system is used for the driving assistance purpose. This System have minimum false ratio so that this can be used in the real time.

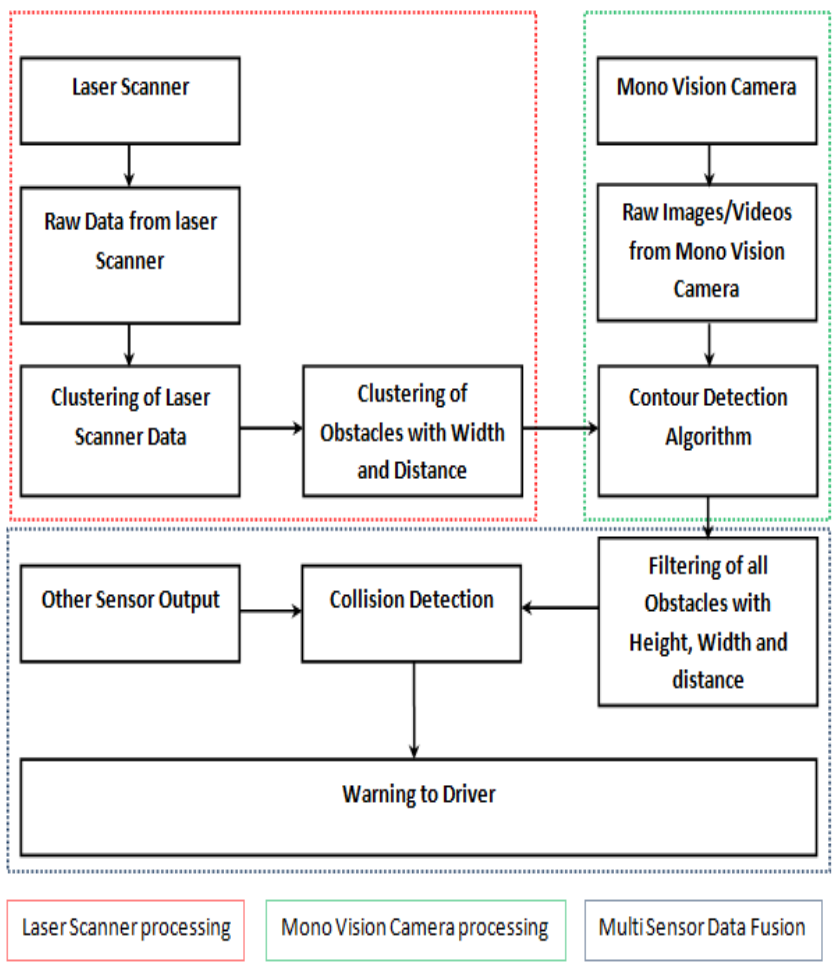

Fig. 2 Architecture of proposed system

\subsection{Laser Scanner Processing}

In Laser Scanner Processing section we get the raw data from the laser scanner. Then we cluster the data given by the laser scanner by clustering algorithm. On that basis we get the width and depth of the obstacles. Some time it may give false data when laser scanner hits on the road, that false data will be confirmed by the mono vision camera processing unit.

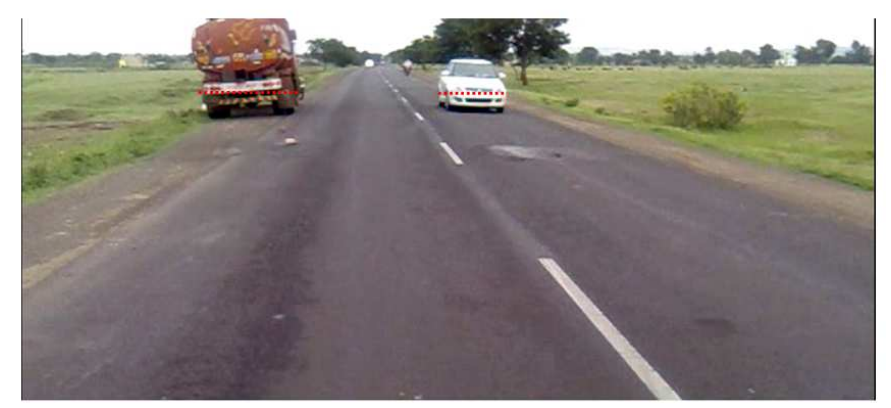

Fig. 3 Laser Scanner processing unit output

\subsection{Mono Vision Camera Processing}

When Laser scanner unit finds any obstacles then mono vision camera processing section will get that image and process that image using contour detection algorithm for finding out the obstacles. Some time laser scanner gives the false data at that time mono vision camera will decline the result of laser scanner only if there is no obstacles founds.

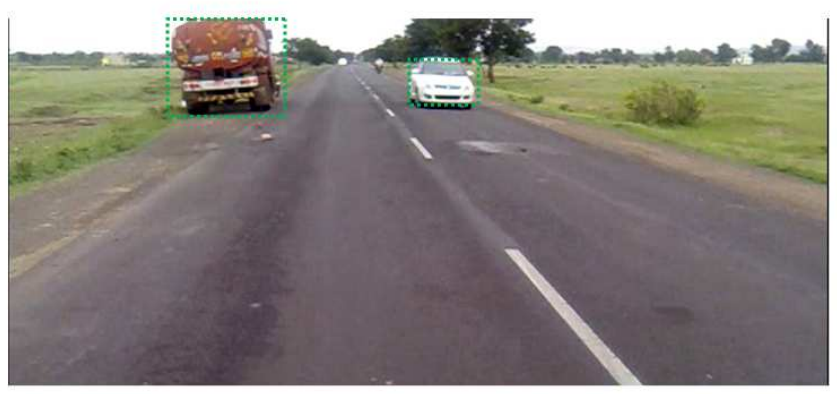

Fig. 4 Mono Vision Camera processing unit output

\subsection{Multi Sensor Data Fusion}

Filtering of obstacles will be done in multi sensor data fusion section. Which will depends on the data given by laser scanner and mono vision camera processing unit. When we find the obstacles then system will calculate the collision between vehicles which is depends on the depth of the obstacles and speed of our vehicle. When collision is done then depending on the distance system will give warning to the driver. 


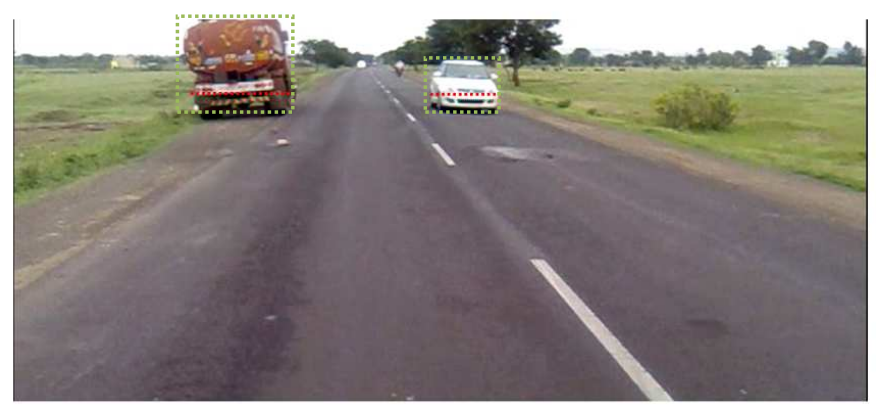

Fig. 5 Multi sensor data fusion output

\section{ALGORITHMS USED}

In this system we used the two main algorithms. These two algorithms represent the two sensor processing. Contour detection algorithm is used for the mono vision camera and clustering algorithm is used for laser scanner.

\subsection{Contour Detection Algorithm}

Contour-based representations have a long history in object recognition and computer vision. Considerable effort was spent in the past matching geometric shape models of objects to image contours. Although these approaches enjoyed some success, it is clear that finding contours exactly belonging to the shape of an object is a hard problem. This insight has given rise to an emphasis on local texture descriptors, the dominant approach today. These appearance-based descriptors summarize local texture information in the form of histograms of gradients, shape context, geometric blur, and many others. While prominent edges are roughly encoded, exact shape location has been replaced by a representation of texture. It is well known, however, that curvature of contours and junctions provide crucial shape information. Thus we believe it is time to investigate a contour representation alongside appearancebased descriptors. In this paper we show that local contour descriptors produce significant gains in object detection and complement texture descriptors. Object contours are a strong representation of shape, whereas texture-based representations summarize contours to avoid matching them to an object exactly. We demonstrate in this work the value of bridging the assets of both. We propose a local contour representation that complements texture features by flexibly encoding junction information and curvature. The representation discretizes contour orientation at an interest point and records the intensity of the contour at each angle as feature elements. Our local contour representation is a non-parametric distribution over oriented line segments, or bars, sampled densely along contours. Recent work has modelled a number of adjacent contour segments to represent shape. The descriptor in this case has a fixed junction degree, utilizes long segments and requires them to be ordered. Other recent contour representations typically break contours into view-dependent segments and assemble them by voting or with constrained splines. The length of the segments in these approaches pushes the descriptor outside the bounds of local information, precluding a simple association with local texture descriptors. We also make a distinction with appearance-based descriptors, such as $\mathrm{HoG}$, that integrate over edge orientations in image regions and produce a texture summary that marginalizes contour detail. In contrast, our oriented bar descriptor defines a distribution over local curvature and junctions at points along contours. The contour representation we introduce is highly repeatable throughout an object category and encodes local curvature information. Object shape contours contain local structure that repeats throughout standard views of a category. The corners of a mug, for example, and the junctions where a handle attaches, appear in most exemplars with some angular variation. For this example we computed oriented bar features over Applelogos of the ETHZ shape dataset and clustered them into 64 groups using kmeans. We observe that much of the object shape can be explained by a combination of these local contours.

In addition to being repeatable, our contour representation describes local curvature. Understanding angle information in contours is important because shape cues concentrate there; segments with high curvature encode more information about shape than straight lines. Where we sampled interest points uniformly over detected edges and computed oriented bar features. We then rendered the features in order of their contour strength and curvature. The latter is measured by the outer product of an oriented bar feature with itself, weighted by angle difference, e.g. $\sum f_{i j} e^{-\left|\theta_{i}^{-}-\theta_{j}\right|}$ We observe that the object is identifiable after rendering a relatively small number of oriented bar features. Curvature clearly concentrates shape information, and the oriented bar descriptor captures this at contour interest points.

We first briefly outline the detection strategy for evaluating the combination of contour and texture information. We evaluate the proposed representation based on the widely used Hough transform for object detection. The approach is kept general to allow for wide applicability rather than specializing on a specific object category. The emphasis is on evaluating local contour properties, not the voting or verification stages. Nonetheless, we found that this simple detector, based on our object representation, is surprisingly effective; it exceeds many state-of-the-art voting-based 
detection systems. To make a detection we first extract edge contours $\mathrm{E}$ from an image using the Berkeley

edge detector. We sample $\mathrm{N}$ interest points locations $\mathrm{X} 1$...........XN uniformly along contours. At each of the locations we compute our oriented bar contour representation $\mathrm{b} 1$...........bN and the texture-based geometric blur features $g 1 \ldots \ldots . . . \mathrm{gN}$. We then concatenate the descriptors at the i-th interest point into a feature vector $\mathrm{fi}=$ (bi, gi).

For each of the features fi in the test image, we find a nearest neighbour fi in training set. We use Euclidean distance for matching and a kd-tree to make the retrieval efficient. We could quantize the features into a codebook, if matching performance becomes an issue. As part of the training process, we store a shift vector vi that points to the object center for each of the training instances fi. Then for matches f1.......fN, we have a corresponding set of a shift vectors $v 1 \ldots \ldots \ldots . . . \mathrm{vN}$. We combine the vi with interest point locations xi to cast votes for the position of an object.

In addition to voting for object position, we cast each of the votes through a set of discrete scales. Formally, we vote for object hypotheses over position and scale space in a Hough accumulator

$$
H(x, \sigma)=\sum_{i=1}^{N} w_{i} \delta\left(\left\|x_{i}+\sigma v_{i}-x\right\|\right),
$$

Where $\delta$ is the Dirac delta function. This equation has been used numerous times and the weights have been estimated in many ways, including probability models, max-margin classifiers, and constrained co-activations. Surprisingly, we found in our experiments that uniform weights exceed the performance of most of these methods (excluding postprocessing verification stages).

We estimate a discrete density over image position and scale space using a 3-D Gaussian

$$
h(x, \sigma)=c \sum_{\mathrm{x}, \mathrm{s}} H(x-\mathrm{x}, \sigma-\mathrm{s}) \eta\left(\mathrm{x}, \mathrm{s} ; \omega_{x}, \omega_{\sigma}\right) .
$$

This is a convolution of the Hough accumulator with a kernel function $\eta$ that has $w_{x}^{2}$ and $w_{\sigma}^{2}$ on the diagonal of its covariance matrix. The constant $\mathrm{c}$ normalizes the discrete density. We can efficiently compute h using the fast Fourier transform. We identify object hypotheses as local maxima according to their 26-way neighbourhood. Watershed segmentation over position in each scale of $h$ gives the features casting votes for a hypothesis.

\subsection{Clustering Algorithm}

$\mathrm{KNN}$ is a simple algorithm to clustering various data based on the similar measures. KNN has been used in statically estimation and pattern recognition. Basic aim of KNN algorithm in the proposed system is to clustering the objects and depending on the objects it will classify different objects and gives the information about clustered objects like $\mathrm{x}$ and $y$ coordinates width of object and depth of object. KNN assumes that the data is in a feature space. More exactly, the data points are in a metric space. The training data provided to KNN algorithm can be scalar or multidimensional vector. Each training data consist of set of vector and associated label with each vector. KNN can work effectively on arbitrary number of classes. We are also given a single number " $k$ ". This number decides how many neighbors (where neighbors are defined based on the distance metric) influence the classification. This is usually an odd number if the number of classes is 2 . If $\mathrm{k}=1$, then the algorithm is simply called the nearest neighbor algorithm.

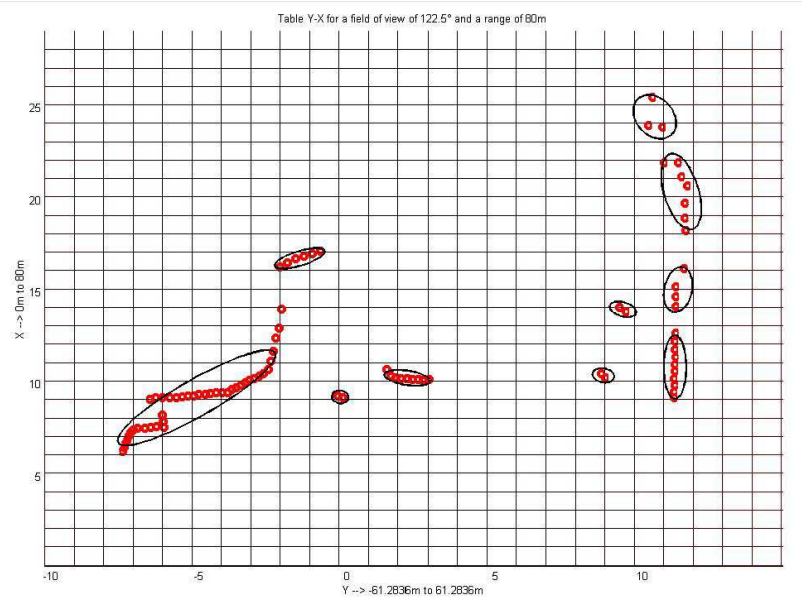

Fig. 6 K-Nearest Neighbor Algorithm (KNN)

\section{CONCLUSIONS}

The proposed system is used to detect the obstacles on the road. This is done by the fusion of raw data from the two sensors which is mono vision camera and laser scanner. In this system laser scanner is used to detect the obstacles and then mono vision camera is used to confirm the obstacles. Depending on the distance system gives the warning to the driver. This system is very helpful for the driving assistance purpose so that decrease the number of accident on the road. This system is best example of cooperative fusion between multiple sensors and depending on that it will take the decision so that the result will be more precise and accurate. 


\section{REFERENCES}

[1]. Raphel Labayrade, Cyril Royere, Dominique Gruyer and Didder Aubert Livic,"Cooperative Fusion for Multi-Obstacles Detection With Use of Stereovision and Laser Scanner", Autonomous Robots 19, 117-140, Springer Science and Business Media, 2009.

[2]. Mathias Perrollaz, Raphael Labayrade, Cyril Royere, Nicolas Hautiere, Didier Aubert, "Long Range Obstacle Detection Using Laser Scanner and Stereovision", published in "IEEE Intelligent Vehicles Symposium (2010)".

[3]. Ho Gi Jung1,, Yun Hee Lee1, Pal Joo Yoon, In Yong Hwang, and Jaihie Kim, "Sensor Fusion Based Obstacle Detection/Classification for Active Pedestrian Protection System", Springer-Verlag Berlin Heidelberg 2009, pp 294-305.

[4]. Takahiro Kuroki, Kenji Terabayashi and Kazunori Umeda. "Construction of a compact range image sensor using multi-slit laser projector and obstacle detection of a humanoid with the sensor", The 2010 IEEE/RSJ International Conference on Intelligent Robots and Systems.October 18-22, 2010, Taipei, Taiwan. Pp 5972-5977

[5]. Mahdi Rezaei Ghahroudi1 and Reza Sabzevari2, "Multisensor Data Fusion Strategies for Advanced Driver Assistance Systems", Open Access Database ISBN 978-3- 902613-52-3, February 2009, pp. 490.

[6]. Neelu Singh, Frost and Sullivan,"Rear obstacle detection systems: a boon for vehicle safety", Nov/Dec 2007 EngineerIT, pp 68-69.

[7]. Dr. Angelos Amditis, Aris Polychronopoulos, Ioannis Karaseitanidis, Dr. George Katsoulis, Multiple - Sensor - Collision avoidance system for automotive applications using an IMM approach for obstacle tracking", ISIF, 2002, pp 812-817

[8]. Dr. Ciza Thomas, "Sensor Fusion and Its Applications", Janeza Trdine 9, 51000 Rijeka, Croatia, 2010, 375-394.

[9]. Stephen Ezell, "Intelligent Transportation Systems", The Information Technology and Innovation Foundation, Jan 2010.

[10]. Sumit Badal Srinivas Ravela Bruce Draper Allen Hanson, "A Practical Obstacle Detection and Avoidance System", ARPA (via TACOM) grant DAAE07-91-C-R035 and NSF grant CDA-8922572. 1998.

[11]. Markus Enzweiler,Dariu M. Gavrila, "Monocular Pedestrian Detection:Survey and
Experiments", IEEE tranc. on pattern analysis and machine intelligence, Oct 2008.

[12]. Cong Yang, Peng Jun-Jian, Sun Jing, Zhu Lin-Lin, Tang Yan- Dong, "V-disparity Based UGV Obstacle Detection in Rough Outdoor Terrain", ACTA AUTOMATICA SINICA, VOl-36 No-5, May 2010, pp 667-673.

[13]. G. Fasano, D. Accardo, L. Forlenza, A. Moccia1, and A. Rispoli, "A MULTI-SENSOR OBSTACLE DETECTION AND TRACKING SYSTEM FOR AUTONOMOUS UAV SENSE AND AVOID”, XX Congresso Nazionale AIDAA Mi- lano, 2009.

[14]. Robert Nowak, Jacek Misiurewicz,Rafa Biedrzycki, "Auto- matic Adaptation in Classification Algorithms Fusing Data From Heterogeneous Sensors", 14th International Conference on Information Fusion Chicago, Illinois, USA, July 5-8, 2011.

[15]. Maria Isabel Ribeiro, "Obstacle Avoidance", Naviga- tion/Collision Avoidance, 3rd November 2005.

[16]. Navneet Dalal and Bill Triggs. Histograms of oriented gradients for human detection. In IEEE Computer Society Conference on Computer Vision and Pattern Recognition, pages 886-893, June 2005.

[17]. Pedro F. Felzenszwalb, Ross B. Girshick, David McAllester, and Deva Ramanan. Object detection with discriminatively trained part based models. IEEE Transactions on Pattern Analysis and Machine Intelligence, 32(9):1627-1645, September 2010.

[18]. Vittorio Ferrari, Loic Fevrier, Frederic Jurie, and Cordelia Schmid. Groups of adjacent contour segments for object detection. IEEE Transactions on Pattern Analysis and Machine Intelligence, 30(1):36-51, January 2008.

[19]. Vittorio Ferrari, Frederic Jurie, and Cordelia Schmid. From images to shape models for object detection. International Journal of Computer Vision, pages 1-20, 2009.

[20]. David R. Martin, Charless Fowlkes, and Jitendra Malik. Learning to detect natural image boundaries using local brightness, color, and texture cues. IEEE Transactions on Pattern Analysis and Machine Intelligence, 26(5):530-549, May 2004.

[21]. Björn Ommer and Jitendra Malik. Multiscale object detection by clustering lines. In International Conference on Computer Vision, pages 484-491, 2009. 
[22]. Andreas Opelt, Axel Pinz, and Andrew Zisserman. Learning an alphabet of shape and appearance for multi-class object detection. International Journal of Computer Vision, 80(1):16-44, 2008.

[23]. Alex P. Pentland. Recognition by parts. In International Conference on Computer Vision, pages 612-620, 1987.

[24]. Xiaofeng Ren, Charless Fowlkes, and Jitendra Malik. Figure/ground assignment in natural images. In European Conference on Computer Vision, volume 2, pages 614-627, 2006.

[25]. Jamie Shotton, Andrew Blake, and Roberto Cipolla. Contour-based learning for object detection In International Conference on Computer Vision, pages 503-510, 2005.

[26]. Praveen Srinivasan, Qihui Zhu, and Jianbo Shi. Many-to-one contour matching for describing and discriminating object shape. In IEEE Computer Society Conference on Computer Vision and Pattern Recognition, pages 1673-1680, 2010. 